\title{
K2-110 b: a massive mini-Neptune exoplanet
}

H. P. Osborn ${ }^{1}$, A. Santerne ${ }^{2,3}$, S. C. C. Barros ${ }^{2}$, N. C. Santos ${ }^{2,4}$, X. Dumusque ${ }^{5}$, L. Malavolta ${ }^{6,7}$, D. J. Armstrong ${ }^{1,8}$, S. Hojjatpanah ${ }^{2,9}$, O. Demangeon ${ }^{3}$, V. Adibekyan ${ }^{2}$, J.-M. Almenara ${ }^{10,11}$, D. Barrado ${ }^{11}$, D. Bayliss ${ }^{5}$, I. Boisse ${ }^{3}$, F. Bouchy ${ }^{5,3}$, D. J. A. Brown ${ }^{1}$, A. C. Cameron ${ }^{12}$, D. Charbonneau ${ }^{13}$, M. Deleuil ${ }^{3}$, E. Delgado Mena ${ }^{2}$, R. F. Díaz ${ }^{5,14,15}$, G. Hébrard ${ }^{16,17}$, J. Kirk ${ }^{1}$, G. W. King ${ }^{1}$, K. W. F. Lam ${ }^{1}$, D. Latham ${ }^{13}$, J. Lillo-Box ${ }^{18}$, T. M. Louden ${ }^{1}$, C. Lovis ${ }^{5}$, M. Marmier ${ }^{5}$, J. McCormac ${ }^{1}$, E. Molinari ${ }^{19,20}$, F. Pepe ${ }^{5}$, D. Pollacco ${ }^{1}$, S. G. Sousa ${ }^{2}$, S. Udry ${ }^{5}$, and S. R. Walker ${ }^{1}$

1 Department of Physics, University of Warwick, Gibbet Hill Road, Coventry, CV4 7AL, UK e-mail: h.p.osborn@warwick.ac.uk

2 Instituto de Astrofísica e Ciências do Espaço, Universidade do Porto, CAUP, rua das Estrelas, 4150-762 Porto, Portugal

3 Aix Marseille Université, CNRS, Laboratoire d'Astrophysique de Marseille UMR 7326, 13388 Marseille, France

${ }^{4}$ Departamento de Física e Astronomia, Universidade do Porto, rua Campo Alegre, 4169-007 Porto, Portugal

5 Observatoire Astronomique de l'Université de Genève, 51 Chemin des Maillettes, 1290 Versoix, Switzerland

6 Dipartimento di Fisica e Astronomia “Galileo Galilei”, Universita' di Padova, Vicolo dell'Osservatorio 3, 35122 Padova, Italy

INAF-Osservatorio Astronomico di Padova, Vicolo dell'Osservatorio 5, 35122 Padova, Italy

8 ARC, School of Mathematics \& Physics, Queen's University Belfast, University Road, Belfast BT7 1NN, UK

9 Department of Physics, University of Zanjan, University Blvd, 45371-38791 Zanjan, Iran

10 Université Grenoble Alpes, IPAG, 38000 Grenoble, France

11 CNRS, IPAG, 38000 Grenoble, France Depto. de Astrofísica, Centro de Astrobiología (CSIC-INTA), ESAC campus 28692 Villanueva de la Cañada (Madrid), Spain

12 School of Physics \& Astronomy, University of St. Andrews, North Haugh, St. Andrews Fife, KY16 9SS, UK

13 Harvard-Smithsonian Center for Astrophysics, 60 Garden Street, Cambridge, Massachusetts 02138, USA

14 Universidad de Buenos Aires, Facultad de Ciencias Exactas y Naturales, Buenos Aires, Argentina

15 CONICET - Universidad de Buenos Aires, Instituto de Astronomía y Física del Espacio (IAFE) Buenos Aires, Argentina

16 Institut d'Astrophysique de Paris, UMR 7095 CNRS, Université Pierre \& Marie Curie, 98bis boulevard Arago, 75014 Paris, France

17 Observatoire de Haute-Provence, Université d'Aix-Marseille \& CNRS, 04870 Saint Michel l'Observatoire, France

18 European Southern Observatory (ESO), Alonso de Cordova 3107, Vitacura, Casilla 19001, Santiago de Chile, Chile

19 INAF - Fundación Galileo Galilei, Rambla José Ana Fernandez Pérez 7, 38712 Berña Baja, Spain

20 INAF - IASF Milano, via Bassini 15, 20133 Milano, Italy

Received 13 May 2016 / Accepted 25 April 2017

\begin{abstract}
We report the discovery of the exoplanet K2-110 b (previously EPIC212521166b) from K2 photometry orbiting in a 13.8637d period around an old, metal-poor K3 dwarf star. With a $V$-band magnitude of 11.9, K2-110 is particularly amenable to RV follow-up. A joint analysis of K2 photometry and high-precision RVs from 28 HARPS and HARPS-N spectra reveal it to have a radius of $2.6 \pm 0.1 R_{\oplus}$ and a mass of $16.7 \pm 3.2 M_{\oplus}$, hence a density of $5.2 \pm 1.2 \mathrm{~g} \mathrm{~cm}^{-3}$, making it one of the most massive planets yet to be found with a sub-Neptune radius. When accounting for compression, the resulting Earth-like density is best fitted by a $0.2 M_{\oplus}$ hydrogen atmosphere over an $16.5 M_{\oplus}$ Earth-like interior, although the planet could also have significant water content. At 0.1 AU, even taking into account the old stellar age of $8 \pm 3 \mathrm{Gyr}$, the planet is unlikely to have been significantly affected by EUV evaporation. However the planet likely disc-migrated to its current position making the lack of a thick $\mathrm{H}_{2}$ atmosphere puzzling. This analysis has made $\mathrm{K} 2-110 \mathrm{~b}$ one of the best-characterised mini-Neptunes with density constrained to less than $30 \%$.
\end{abstract}

Key words. planets and satellites: detection

\section{Introduction}

Since 2014, Kepler's extended K2 mission (Howell et al. 2014) has observed eleven 80-day fields, giving precise photometry for more than 200000 stars. So far, it has produced around 500 planet candidates (Foreman-Mackey et al. 2015; Vanderburg et al. 2016; Barros et al. 2016; Pope et al. 2016) and nearly 150 confirmed or validated planets (Montet et al. 2015; Sinukoff et al. 2016; Armstrong et al. 2015b; Barros et al. 2015; Crossfield et al. 2016). K2 has also significantly expanded the population of small planets transiting bright stars, with the number of K2 planets around stars with Kepler magnitude
11-12.5 already exceeding the initial four-year Kepler mission (Crossfield et al. 2016).

Large super-Earths and mini-Neptunes are an interesting and diverse population to study. Lying in the transition regime between terrestrial planets and gas giants, they can provide direct constraints on giant planet formation theory (e.g. Hansen \& Murray 2012). They range from low-density neptunes (e.g. the Kepler-11 system; 0.6-1.7 $\mathrm{g} \mathrm{cm}^{-3}$, Lissauer et al. 2013), to super-Earths with large rocky interiors (e.g. Kepler$10 \mathrm{c} ; 7 \pm 1 \mathrm{~g} \mathrm{~cm}^{-3}$ Dumusque et al. 2014), to controversial claims of ultra-dense super-Earths that could be evaporated gas giant cores (e.g. K2-38 b; $17.5 \pm 7 \mathrm{~g} \mathrm{~cm}^{-3}$ Sinukoff et al. 2016). 


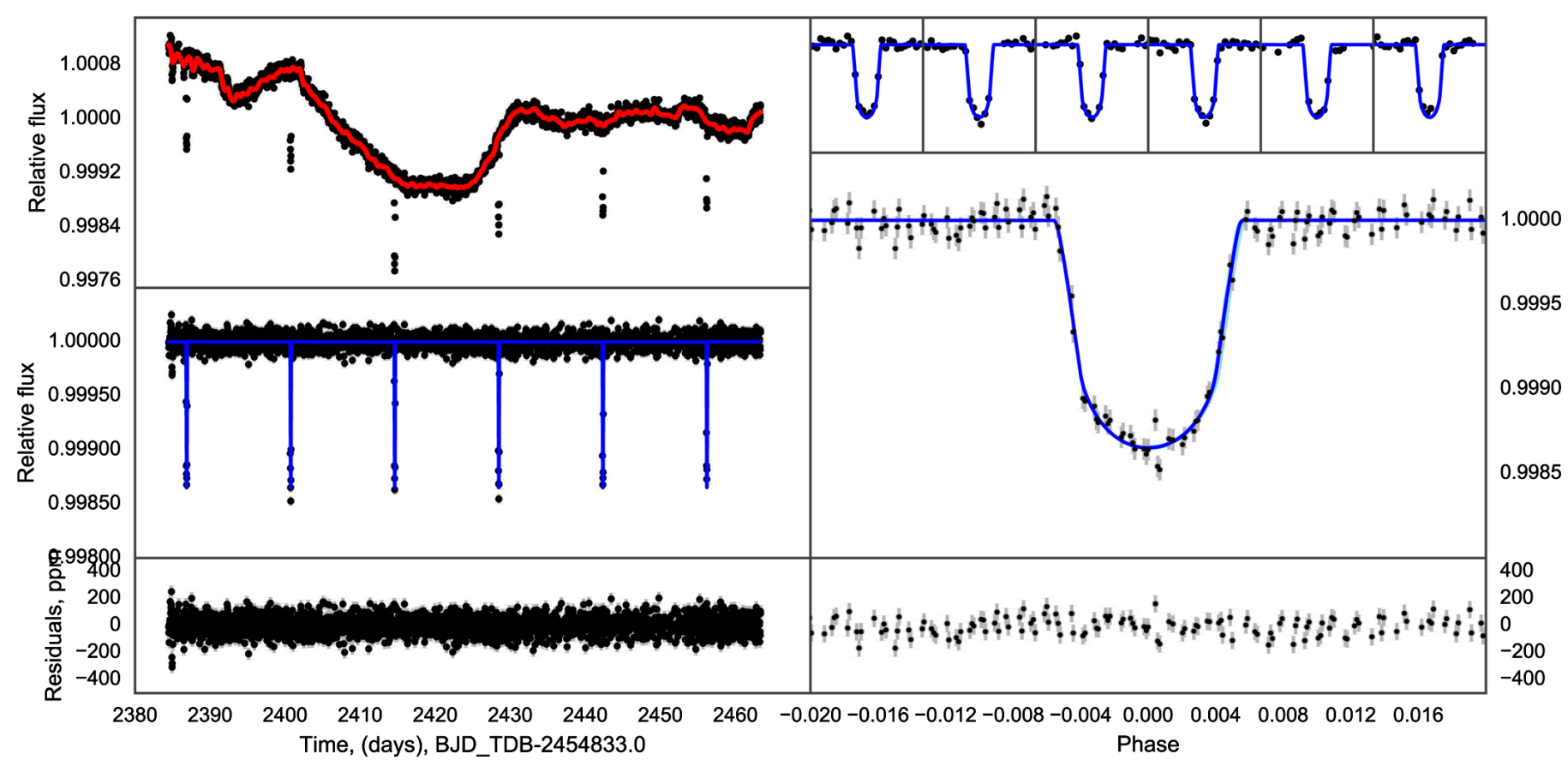

Fig. 1. Transit lightcurve and model best-fit. Detrended K2 lightcurve (upper left); lightcurve smoothed with Gaussian Processes (centre left); bestfit transit model lightcurve residuals (lower left); all six transits (upper right); phase-folded unbinned lightcurve centred on transit with best-fit model in blue and best-fit region in light blue (centre right); phase-folded model residuals (lower right).

With magnitudes brighter than $V=12$, such planets are also suitable targets for ground-based follow-up targeting radial velocities (RVs), the Rossiter-McLaughlin effect and atmospheric spectroscopy. We present the detection of a sub-Neptune radius planet around the $\mathrm{K}$ field dwarf K2-110 from K2 photometry and the confirmation and mass measurement of this planet with HARPS and HARPS-N RVs.

\section{Observations, data reduction and analysis}

\subsection{K2 Photometry}

EPIC212521166 was observed during Campaign 6 of the K2 mission. We downloaded the pixel data from the Mikulski Archive for Space Telescopes (MAST) ${ }^{1}$ and used a modified version of the CoRoT imagette pipeline to extract the light curve. Based on signal-to-noise of each pixel we computed an optimal aperture of 25 pixels. The background was estimated using the $3 \sigma$ clipped median of all the pixels in the image and subtracted. We also calculated the centroid using the Modified Moment Method by Stone (1989). In order to correct for flux variations due to the star's apparent motion on the charge-coupled device (CCD) we used a self-flat-fielding procedure similar to Vanderburg \& Johnson (2014). This assumes the movement of the satellite was mainly in one direction, as described in Barros et al. (2016). The lightcurve was flattened with an exponential square Gaussian process trained on out-of-transit data. Transit fitting was performed jointly with RVs and is described in Sect. 2.4. The final light curve of K2-110 has mean out-oftransit RMS of 134 parts per million (ppm) per 30 min cadence.

\footnotetext{
1 http://archive.stsci . edu/kepler/data_search/search. php
}

\subsection{Detection}

EPIC212521166 was indentified as a strong planet candidate during two independent searches for transits in the lightcurve. Both Barros et al. (2016) using the CoRoT alarm pipeline and the detrending method defined above, and a manual search of bright Campaign 6 lightcurves detrended using the technique of Armstrong et al. (2015a) detected K2-110 b. It was also independently identified by Aigrain et al. (2016) \& Pope et al. (2016) although no detailed analysis was performed.

\subsection{Radial velocity follow-up}

We performed RV follow-up of the target star. A single exposure with the CORALIE spectrograph (Queloz et al. 2000) mounted on the EULER telescope at ESO La Silla observatory, Chile confirmed that the target was suitable for precise radial velocities.

The target was then observed with the HARPS spectrograph (Mayor et al. 2003), mounted on the $3.6 \mathrm{~m}$ telescope at ESO La Silla observatory ${ }^{2}$. Seventeen exposures of $3600 \mathrm{~s}$ in the obj_AB mode were secured on 13 nights from 2016-03-03 to 2016-0810 , with $\mathrm{S} / \mathrm{N}$ per pixel at $5500 \AA$ from 25 to 57 leading to photon noise uncertainty $\left(\sigma_{\mathrm{RV}}\right)$ in the range $1.2-3.0 \mathrm{~m} \mathrm{~s}^{-1}$.

Eleven further spectra were also taken with the HARPS-N spectrograph (Cosentino et al. 2012), mounted on the $3.58 \mathrm{~m}$ Telescopio Nazionale Galileo at ING La Palma observatory. These had a median exposure time of 1800s each and were secured on 11 nights from 2016-05-12 to 2016-06-25, with S/N per pixel at order $n=50$ (5500 $\AA$ ) from 15 to 37 . The HARPS-N exposures suffered from contamination of moonlight, which were corrected by subtracting the CCF of the spectrum of the sky (gathered with fiber B) from the CCF of the target star (gathered with fiber A), after recomputing the former with the same flux correction coefficients of the latter (see Malavolta et al. 2017, for

\footnotetext{
2 ESO programme ID: 096.C-0657.
} 


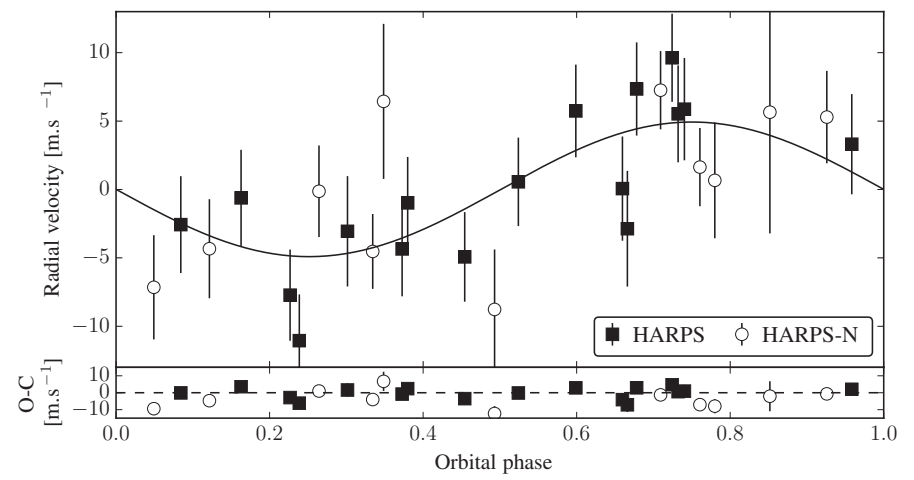

Fig. 2. RVs taken by HARPS and HARPS-N, phased to the $13.86 \mathrm{~d}$ period as determined from the K2 lightcurve. The best model is also displayed together with the residuals.

details). Although contamination corrections were applied to all HARPS-N measurements, only six of these had absolute RV corrections $(|\Delta \mathrm{RV}|)$ greater than the photon noise $\left(\sigma_{\mathrm{RV}}\right)$, with four being corrected by more than $2 \sigma_{\mathrm{RV}}$ (see Table 4 ).

We computed the RVs from each high-resolution spectrum using weighted cross-correlation with a K5 template (Baranne et al. 1996; Pepe et al. 2002) as implemented by the HARPS and HARPS-N pipelines. RV uncertainties were determined as described in Bouchy et al. (2001). They range from 1.1 to $8.8 \mathrm{~m} \mathrm{~s}^{-1}$. The pipeline also computed the averaged line profile full-width half maximum (FWHM), bisector span and the $\log R_{\mathrm{HK}}^{\prime}$. All these data are reported with uncertainties in Table A.1.

\subsection{Host star parameters}

Stellar atmospheric parameters and $[\mathrm{Fe} / \mathrm{H}]$ were derived in LTE using a recent version of the MOOG code (Sneden 1973) and a set of plane-parallel ATLAS9 model atmospheres (Kurucz 1993), as described in Sousa et al. (2011). The full spectroscopic analysis is based on the equivalent widths (EWs) of $103 \mathrm{Fe} I$ and $15 \mathrm{Fe}$ II weak lines by imposing ionization and excitation equilibrium. The line-list used was taken from Tsantaki et al. (2013), and is adapted for stars with $T_{\text {eff }}<5200 \mathrm{~K}$. The stellar parameters derived using this methodology were shown to be in line with the ones derived using other methods, for example, interferometry and infra-red flux method (IRFM) (see Santos et al. 2013 , for details). Our derived values of $T_{\text {eff }}(4960 \pm 60 \mathrm{~K})$, $\log (g)(4.58 \pm 0.13)$ and $[\mathrm{Fe} / \mathrm{H}](-0.34 \pm 0.03)$ are used as priors in PASTIS (see Sect. 2.5 and Table 5) and therefore are re-derived in a self-consistent manner with all observed stellar information. We also find a $\log R_{\mathrm{HK}}^{\prime}$ of $-4.983 \pm 0.002$. Chemical abundances for different species derived using the methodology described in, for example Adibekyan et al. (2016) and Santos et al. (2015) are shown in Table A.2.

\subsection{PASTIS analysis}

We jointly analysed the HARPS/HARPS-N RVs, K2 photometric light curve within $3 \mathrm{~h}$ of the transit midtime and the spectral energy distribution (SED) as observed by the APASS, 2-MASS and WISE surveys (Munari et al. 2014; Cutri et al. 2014) using the PASTIS software (Díaz et al. 2014; Santerne et al. 2015). It models the light curve using the jktebop package (Southworth 2008) assuming an oversampling factor (Kipping 2010) of 30 to account for the long integration time of the K2 data. The SED
Table 1. Stellar Information for K2-110. Magnitudes from ExoFOP-K2.

\begin{tabular}{lc}
\hline \hline Parameter & Value and uncertainty \\
\hline Stellar information & \\
& \\
EPIC & 212521166 \\
RA & $13^{\mathrm{h}} 49^{\mathrm{m}} 23.890^{\mathrm{s}}$ \\
Dec & $-12^{\mathrm{d}} 17^{\mathrm{m}} 04.16^{\mathrm{s}}$ \\
$2 \mathrm{MASS}$ ID & $2 \mathrm{MASS} \mathrm{J} 13492388-1217042$ \\
$\mu_{\text {RA }}$ & $42.6 \pm 1.1 \mathrm{mas} / \mathrm{yr}$ \\
$\mu_{\text {Dec }}$ & $-101.2 \pm 1.4 \mathrm{mas} / \mathrm{yr}$ \\
\end{tabular}

Photometric magnitudes

\begin{tabular}{lc}
$B$ & $12.834 \pm 0.05$ \\
$V$ & $11.91 \pm 0.07$ \\
Kep & 11.59 \\
$J$ & $10.184 \pm 0.022$ \\
$H$ & $9.641 \pm 0.023$ \\
$K$ & $9.607 \pm 0.024$ \\
WISE $3.4 \mu \mathrm{m}$ & $9.521 \pm 0.024$ \\
WISE $4.6 \mu \mathrm{m}$ & $9.577 \pm 0.020$ \\
WISE $12 \mu \mathrm{m}$ & $9.479 \pm 0.038$ \\
WISE $22 \mu \mathrm{m}$ & 8.695 \\
\hline
\end{tabular}

was modelled using the BT-SETTL library of stellar atmosphere (Allard et al. 2012). The RVs were modelled with a Keplerian orbit. A Markov chain Monte Carlo (MCMC) method was used to analyse the data. The results from the spectral analysis described in Sect. 2.4 were used as priors for the host star. The spectroscopic parameters were converted into fundamental stellar parameters in the MCMC using the Darthmouth evolution tracks (Dotter et al. 2008). For detail on the priors used see Table 5.

We used uninformative priors for most of the parameters. Exception include the stellar atmospheric parameters, for which we used the inputs of Sect. 2.4; the orbital eccentricity, for which we choose a Beta distribution (Kipping 2013); and the orbital ephemeris, for which we choose uniform distributions centred on the values found by the detection pipeline.

We ran 20 independent MCMCs of $3 \times 10^{5}$ iteration randomly started from the joint prior distribution. We then removed the burn-in phase before merging the converged chains (see Díaz et al. 2014). The residuals of the RV have a RMS at the level of $3.0 \mathrm{~m} \mathrm{~s}^{-1}$, which is approximately twice the median photon noise. The fit was also run with uniform priors on both eccentricity and limb darkening parameters to test if these may be influencing the resulting planet parameters. However, all outputs were consistent within 1-sigma to those obtained during the initial PASTIS fit. Complete stellar and planetary outputs of PASTIS are reported in Table 2.

\section{Discussion}

\subsection{Validity}

The presence of a RV signal in-phase with (and at a similar amplitude to) that expected from the transit detection is extremely strong evidence for a planet. However, we also performed additional tests to ensure that the signal was not due to, for example, a blended eclipsing binary.

Using the cross-correlation function with which we computed RVs, we can exclude to 3sigma all secondaries with $\Delta$ mag $<6.5$, assuming the companion is spectrally resolved (i.e. 
Table 2. Physical parameters of the K2-110 system.

\begin{tabular}{lc}
\hline \hline Parameter & Value and uncertainty \\
\hline Stellar parameters & \\
Stellar mass $M_{\star}\left[M_{\odot}\right]$ & $0.738 \pm 0.018$ \\
Stellar radius $R_{\star}\left[R_{\odot}\right]$ & $0.713 \pm 0.020$ \\
Stellar age $\tau[\mathrm{Gyr}]$ & $8 \pm 3$ \\
Distance $d[\mathrm{pc}]$ & $118.0 \pm 3.6$ \\
Reddening $E(B-V)[\mathrm{mag}]$ & $0.019_{-0.013}^{+0.019}$ \\
Systemic $\mathrm{RV} v_{0}\left[\mathrm{~km} \mathrm{~s}^{-1}\right]$ & $-21.6331 \pm 9 \times 10^{-4}$ \\
Effective temperature $T_{\mathrm{eff}}[\mathrm{K}]$ & $5010 \pm 50$ \\
Surface gravity $\log g\left[\mathrm{~g} \mathrm{~cm}{ }^{-2}\right]$ & $4.60 \pm 0.03$ \\
Iron abundance $[\mathrm{Fe} / \mathrm{H}][\mathrm{dex}]$ & $-0.34 \pm 0.03$ \\
Spectral type & $\mathrm{K} 3 \mathrm{~V}$ \\
&
\end{tabular}

\section{Orbital parameters}

Period $P[\mathrm{~d}]$

Transit epoch $T_{0}$ [BJD $\left.\mathrm{BDB}_{\mathrm{TD}}\right]$

Orbital eccentricity $e$

Argument of periastron $\omega\left[^{\circ}\right]$

Inclination $i\left[^{\circ}\right]$

Semi-major axis $a$ [AU]

$$
\begin{gathered}
13.86375 \pm 2.6 \times 10^{-4} \\
2457275.32992 \pm 6.1 \times 10^{-4} \\
0.079 \pm 0.07 \\
90_{-64}^{+180} \\
89.35_{-0.24}^{+0.41} \\
0.1021 \pm 8 \times 10^{-4}
\end{gathered}
$$

\section{Transit and radial velocity parameters}

System scale $a / R_{\star}$

Impact parameter $b$

Transit duration $T_{14}$ [h]

Planet-to-star radius ratio $k_{r}$

Limb darkening $u_{a}$

Limb darkening $u_{b}$

RV amplitude $K\left[\mathrm{~m} . \mathrm{s}^{-1}\right]$

HARPS-N RV jitter [ $\mathrm{m} \mathrm{s}^{-1}$ ]

HARPS RV jitter $\left[\mathrm{m} \mathrm{s}^{-1}\right]$

Instrument offset $\left[\mathrm{m} \mathrm{s}^{-1}\right]$

$\mathrm{K} 2$ contamination [flux, ppt]

K2 jitter [flux, ppm]

SED jitter [mag]

$$
\begin{gathered}
30.8 \pm 1.0 \\
0.34_{-0.22}^{+0.14} \\
3.22 \pm 0.03 \\
0.0333 \pm 6.6 \times 10^{-4} \\
0.5322 \pm 1.2 \times 10^{-2} \\
0.1787 \pm 8 \times 10^{-3} \\
5.5 \pm 1.1 \\
3 \pm 2 \\
3.1 \pm 1 \\
4.2 \pm 1.8 \\
3.4_{-2}^{+4} \\
40 \pm 4.6 \\
0.02 \pm 0.02
\end{gathered}
$$

\section{Planet parameters}

Planet mass $M_{\mathrm{p}}\left[M_{\oplus}\right]$

Planet radius $R_{\mathrm{p}}\left[R_{\oplus}\right]$

Planet density $\rho_{\mathrm{p}}\left[\mathrm{g} \mathrm{cm}^{-3}\right]$

Equilibrium temperature $T_{\mathrm{eq}}[\mathrm{K}]$

Notes. All the uncertainties provided here are only the statistical ones. Errors on the models are not considered, as they are unknown. Stellar parameters are derived from the combined analysis of the data and not from the spectral analysis. We assumed $R_{\odot}=695508 \mathrm{~km}$, $M_{\odot}=1.98842 \times 10^{30} \mathrm{~kg}, R_{\oplus}=6378137 \mathrm{~m}, M_{\oplus}=5.9736 \times 10^{24} \mathrm{~kg}$, and $1 \mathrm{AU}=149597870.7 \mathrm{~km}$.

$v_{0}>2.7 \mathrm{~km} \mathrm{~s}^{-1}$ ), and has a similar Teff, metallicity, and rotation (Santerne et al. 2015). Although K2's pixel drift is significant, we can constrain in-transit centroid shifts that result from a signal from a background star to $0.86^{\prime \prime}$ and $0.28^{\prime \prime}$ (2-sigma limits) in the $x$ and $y$ directions. The BIS and FWHM do not exhibit significant variation with RMS at the level of $6 \mathrm{~m} \mathrm{~s}^{-1}$ and $5 \mathrm{~m} \mathrm{~s}^{-1}$, respectively. No significant correlation is found between these spectroscopic diagnoses and the radial velocity with a Pearson test of $0.01 \pm 0.02$, and $0.13 \pm 0.02$, respectively. This strongly supports the planetary nature of the detection (Santerne et al. 2015). Small Neptunes also have the lowest false positive rates (6.7\% in Kepler, Fressin et al. 2013). Together all these are extremely good evidence that the signal is planetary rather than from a false positive, and enables us to designate this planet as confirmed.

\subsection{Age and rotation}

We derived a stellar age of $8 \pm 5$ Gyr from the joint orbital analysis of RVs, photometry, and stellar evolution tracks, with posterior samples cut such that age $<13.5 \mathrm{Gyr}$. No clear rotational signal is detected in the lightcurve, although variation on the order of weeks is seen which could be suggestive of slow starspot rotation (and therefore an old gyrochronological age). A slow stellar rotation and old age are also supported by the upper limit of $v_{\text {rot }}$ measured from HARPS spectra $\left(<2.7 \mathrm{~km} \mathrm{~s}^{-1}, P_{\min }>9.2 \mathrm{~d}\right)$, and the non-detection of lithium $([\mathrm{Li} / \mathrm{H}]<0.2)$.

Recently, Nissen (2015) showed that $[\mathrm{Y} / \mathrm{Mg}]$ ratio can be used to estimate stellar ages. This result was later confirmed by Tucci Maia et al. (2016), the age relation from which suggests an age of $8.1 \pm 2.8 \mathrm{Gyr}$ for K2-110. Hence, all three methods point this star being an old field star, which may also explain its low metallicity.

\subsection{Stellar composition}

The low metallicity of K2-110 is in agreement with the finding that small planets can form around metal-poor host stars (Buchhave et al. 2012). However, the interior mass (16.5 $M_{\oplus}$ ) appears anomalously large for such a metal-poor star, with the median mass sitting above the mass-metallicity upper boundary as found by Courcol et al. (2016) for Neptunian exoplanets. Unusually, K2-110 does not show an enhancement in $\alpha$-elements (e.g. $\mathrm{Si}, \mathrm{Mg}$, etc.) compared to other metal-poor planet hosts (Adibekyan et al. 2012) though it does show enhancement in the pure $\alpha$-element oxygen $([\mathrm{O} / \mathrm{Fe}]=0.35)$.

\subsection{TTVs and other planets}

Using a transit model generated from our PASTIS best fit, we searched for transit timing variations by iteratively shifting the flux model over each individual transit with a resolution of $2.6 \mathrm{~s}$. We detect no significant TTVs and are able to rule out their presence above an amplitude of $6 \mathrm{~min}$ to $3 \sigma$.

We also searched for potential other transiting planets in the system but found no significant signal. Injection and retrieval tests enable us to rule out to $>90 \%$ confidence the presence coplanar planets with orbits $<30 \mathrm{~d}$ and radii $>1 R_{\oplus}$. This, along with the location of K2-110 b at the metallicity-mass upper limit, suggests that the planet is likely solitary and contains the majority of K2-110's protoplanetary disc mass.

\subsection{Composition and formation}

With a mass of $16.7 \pm 3.2 M_{\oplus}$ and a radius of $2.6 \pm 0.1 R_{\oplus}$, this planet stands out as being one of the most massive exoplanets with a sub-Neptune radius (Fig. 3) detected so far. Despite an Earth-like density of $5.2 \pm 1.2 \mathrm{~g} \mathrm{~cm}^{-3}$, a 2-layer iron-silicate composition model is unable to explain the density of K2-110 b. Instead, either low-density volatiles such as 


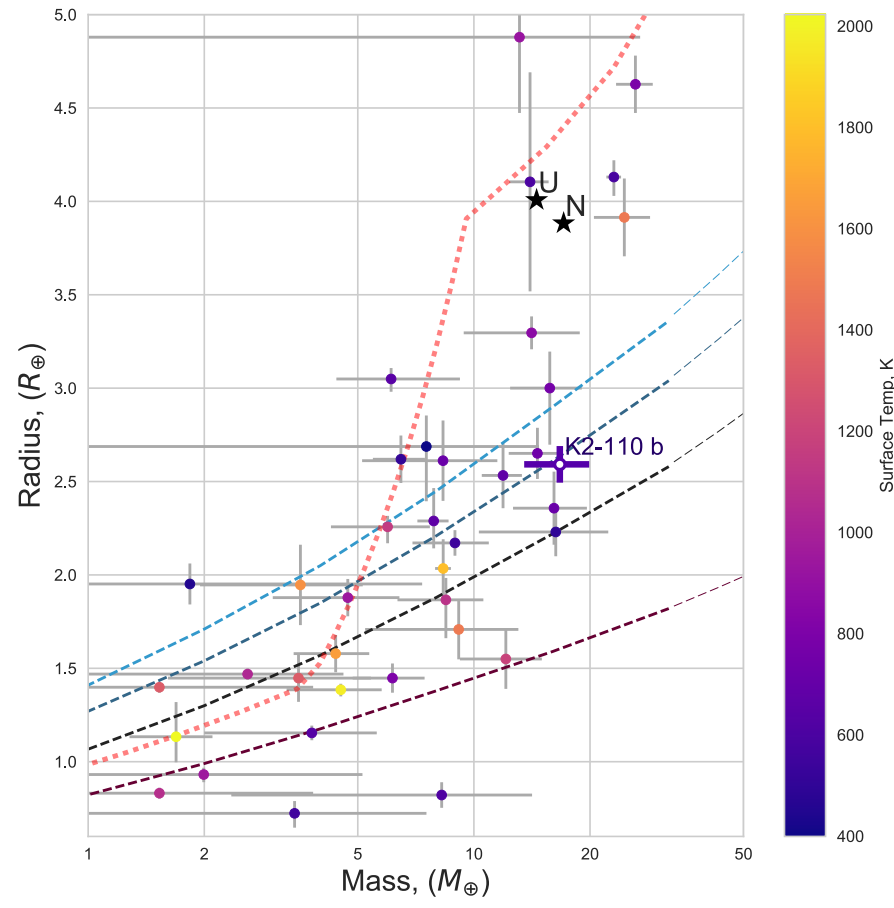

Fig. 3. K2-110 b (solid cross, right) compared to other super-Earth and Neptunian planets (data from Han et al. 2014; Marcy et al. 2014). Mass-radius relations adapted from Zeng \& Sasselov (2013) for 100\%Fe, Earth-like, 50\%-water and 100\% water compositions (dashed lines from bottom to top). A mass-radius relation for small exoplanets (Weiss \& Marcy 2014) is also show (fine dashes).

water, an H-He atmosphere, or a combination of both must be present. We explore the possibility of these degenerate compositions here. Using the 3-layer solid exoplanet composition model of Zeng \& Sasselov $(2013)^{3}$, we compute that a $8.5 M_{\oplus}$ Earthlike interior of $\sim 70 \% \mathrm{MgSiO}_{3}$ and $\sim 30 \%$ Fe covered by $8 M_{\oplus}$ of $\mathrm{H}_{2} \mathrm{O}$ can explain the mass and radius of K2-110 b. As well as surface molecular water, such a model would also require high-pressure water phases Ice VII, Ice $\mathrm{X}$ and superionic fluid (Zeng \& Sasselov 2014).

In the alternate case, the models of Adams et al. (2008) and Lopez \& Fortney (2014) both show that an earth-like $16.5 M_{\oplus}$ interior of iron core and silicate mantle can host a $\sim 0.2 M_{\oplus}$, $\sim 0.4 R_{\oplus} \mathrm{H}-\mathrm{He}$ atmosphere to produce the equivalent bulk density. Intermediate compositions between these two boundary models are also possible. This suggests the mass fraction of hydrogen is likely $<1 \%$ for $\mathrm{K} 2-110 \mathrm{~b}$.

The growing population of high-density planets in the regime from 10 to $20 M_{\oplus}$ also suggests that planets can exist in this region without accreting significant hydrogen. This may therefore suggest that the minimum core accretion mass of $\sim 10 M_{\oplus}$ (Pollack et al. 1996) is underestimated, or that processes exist to remove gaseous atmospheres post-accretion. The unusual density of this planet also suggests that mass-radius relations (e.g. Weiss \& Marcy 2014), should be used with extreme caution in the regime between terrestrial planets and gas giants (e.g. Fig. 3).

To investigate whether K2-110 b could have been formed with a substantial atmosphere that later evaporated, we looked at the potential mass loss from EUV. Taking an average of the most EUV-luminous K-type star, we use the calculations of Lecavelier des Étangs (2007) and calculate a mass loss rate of $2 \times 10^{9} \mathrm{~g} \mathrm{~s}^{-1}$ (Eq. (15)), which corresponds to an upper limit of

\footnotetext{
Accessed from https://www.cfa.harvard.edu/ lzeng/
}

$0.13 M_{\oplus}$ over $10 \mathrm{Gyr}$. This is of similar magnitude to a maximum X-ray mass-loss rate computed from Owen \& Jackson (2012). Therefore, using the Hydrogen atmosphere models mentioned previously, the host star is likely incapable of evaporating more than $0.2 R_{\oplus}$ of Hydrogen from K2-110 b. Thus evaporation is unlikely to have contributed to the high density we see today. Hence K2-110 b likely formed dense, potentially after the gas disc has been photoevaporated.

However, to form $\mathrm{K} 2-110 \mathrm{~b}$ in situ at $0.1 \mathrm{AU}$ would require a disc mass enhanced by 50 compared to solar values (Schlichting 2014). Hence, either material from more distant parts of the disc migrated inwards to build K2-110 b (e.g. Chatterjee \& Tan 2013; Hansen \& Murray 2012), or the planet itself formed far out in the disc and migrated inwards (e.g. McNeil \& Nelson 2010; Kley \& Nelson 2012).

In the latter case, migration could have occurred through dynamic scattering, or through disc migration. Better constraints on orbital eccentricity ( $e=0.08 \pm 0.07$ in this analysis) could help point such an orbital migration mechanism, however we can rule out high eccentricities $(e>0.25)$ typical of warm exoplanets caused by Kozai migration (Dawson \& Chiang 2014). On its relatively wide orbit of $14 \mathrm{~d}, \mathrm{~K} 2-110 \mathrm{~b}$ is also unlikely to have been to acted on by stellar tidal forces; a necessary component of dynamic migration (Fabrycky \& Tremaine 2007). Therefore, if K2-110 b migrated to its current position low-eccentricity disc migration is more likely. However, the lack of a thick $\mathrm{H}$ atmosphere on $\mathrm{K} 2-110 \mathrm{~b}$ is at odds with the migration of a $>10 M_{\oplus}$ planet through a gaseous disc.

On the other hand, models of the migration, impact and accretion of systems of compact planets or planetary embryos (Ida \& Lin 2008; Boley et al. 2016) are able to explain both K2110 b's orbit and its lack of significant hydrogen atmosphere (Liu et al. 2015). Improved orbital parameter measurements (e.g. misalignment and eccentricity) and statistical analyses of exoplanet populations could disentangle which scenario occurred.

\section{Conclusion}

Using photometry from $\mathrm{K} 2$ we have detected a $2.6 \pm 0.1 R_{\oplus}$ planet orbiting an early K-dwarf. RV observations with HARPS and HARPS-N have confirmed K2-110 b (EPIC 212521166) as a planet and measured its mass to be $16.7 \pm 3.2 M_{\oplus}$. The corresponding bulk density suggests $\mathrm{K} 2-110 \mathrm{~b}$ has a large rocky interior and is hydrogen-poor, with $<1 \%$ of its mass in a hydrogen atmosphere. Alternatively, the planet could be volatile-rich, with up to $9 M_{\oplus}$ of $\mathrm{H}_{2} \mathrm{O}$. Our analysis means $\mathrm{K} 2-110 \mathrm{~b}$ is now one of the best-characterised sub-Neptune planets with a radius and mass constrained to $4 \%$ and $20 \%$ respectively. Future observations will improve our understanding of the bulk composition and migration of this planet.

Acknowledgements. We are grateful to the pool of HARPS observers who conducted part of the visitor-mode observations at La Silla Observatory: Fatemeh Motalebi, Aurélien Wyttenbach, Baptiste LaVie, Pedro Figueira, Alessandro Sozzetti, and Anaël Wunsche. The Portuguese team acknowledges the support from the Fundação para a Ciência e Tecnologia (FCT) through national funds and by FEDER through COMPETE2020 by grants UID/FIS/04434/2013 and POCI01-0145-FEDER-007672, PTDC/FIS-AST/1526/2014 and POCI-01-0145FEDER-016886 and PTDC/FIS-AST/7073/2014 and POCI-01-0145-FEDER016880. S.C.C.B., N.C.S. and S.G.S. also acknowledge support from FCT through Investigador FCT contracts numbers IF/01312/2014/CP1215/CT0004, IF/00169/2012/CP0150/CT0002 and IF/00028/2014/CP1215/CT0002. V.Zh.A. and E.D.M. also acknowledge support from FCT through Investigador FCT contracts IF/00650/2015/CP1273/CT0001, IF/00849/2015/CP1273/CT0003, and by the fellowship SFRH/BPD/70574/2010, SFRH/BPD/76606/2011 funded by FCT (Portugal) and POPH/FSE (EC). S.H. acknowledges support by the fellowship PD/BD/128119/2016 funded by FCT (Portugal) and POPH/FSE (EC). A.S. 
was supported by the EU under a Marie Curie Intra-European Fellowship for Career Developmentwith reference FP7-PEOPLE-2013-IEF, number 627202. A.S. is supported by the EU under a Marie Curie Intra-European Fellowship for Career Development with reference FP7-PEOPLE-2013-IEF, number 627202. D.J.A.B. acknowledges support from the UKSA and the University of Warwick. J.L.-B. acknowledges support from the Marie Curie Actions of the European Commission (FP7-COFUND) J.M.A. acknowledges funding from the European Research Council under the ERC Grant Agreement No. 337591-ExTrA. D.J.A. and D.P. acknowledge funding from the European Union Seventh Framework programme (FP7/2007-2013) under grant agreement No. 313014 (ETAEARTH). O.D. acknowledges support by CNES through contract 567133. P.A.W. acknowledges the support of the French Agence Nationale de la Recherche (ANR), under programme ANR-12-BS05-0012 Exo-Atmos. Some of the data presented in this paper were obtained from the Mikulski Archive for Space Telescopes (MAST). STScI is operated by the Association of Universities for Research in Astronomy, Inc., under NASA contract NAS5-26555. Support for MAST is provided by NASA grant NNX09AF08G and by other grants and contracts. This paper includes data collected by the Kepler mission. Funding for the Kepler mission is provided by the NASA Science Mission directorate.

\section{References}

Adams, E., Seager, S., \& Elkins-Tanton, L. 2008, ApJ, 673, 1160 Adibekyan, V. Z., Santos, N. C., Sousa, S. G., et al. 2012, A\&A, 543, A89 Adibekyan, V., Delgado-Mena, E., Figueira, P., et al. 2016, A\&A, 591, A34 Aigrain, S., Parviainen, H., \& Pope, B. 2016, EPIC212521166: a transiting planet candidate discovered in Campaign 6 data from the K2 Mission

Allard, F., Homeier, D., \& Freytag, B. 2012, Phil. Trans. R. Soc. London, Ser. A: Mathematical, Physical and Engineering Sciences, 370, 2765

Armstrong, D., Kirk, J., Lam, K., et al. 2015a, A\&A, 579, A19

Armstrong, D. J., Veras, D., Barros, S. C., et al. 2015b, A\&A, 582, A33

Baranne, A., Queloz, D., Mayor, M., et al. 1996, A\&AS, 119, 373

Barros, S., Almenara, J., Demangeon, O., et al. 2015, MNRAS, 454, 4267

Barros, S., Demangeon, O., \& Deleuil, M. 2016, A\&A, 594, A100

Boley, A., Contreras, A., \& Gladman, B. 2016, ApJ, 817, L17

Bouchy, F., Pepe, F., \& Queloz, D. 2001, A\&A, 374, 733

Buchhave, L. A., Latham, D. W., Johansen, A., et al. 2012, Nature, 486, 375

Chatterjee, S., \& Tan, J. C. 2013, ApJ, 780, 53

Cosentino, R., Lovis, C., Pepe, F., et al. 2012, In Proc. SPIE, 8446, 84461V

Courcol, B., Bouchy, F., \& Deleuil, M. 2016, MNRAS, 461, 1841

Crossfield, I. J., Ciardi, D. R., Petigura, E. A., et al. 2016, ApJS, 226, 7

Cutri, R., et al. 2014, VizieR Online Data Catalog: II/328

Dawson, R. I., \& Chiang, E. 2014, Science, 346, 212

Díaz, R. F., Almenara, J. M., Santerne, A., et al. 2014, MNRAS, 441, 983

Dotter, A., Chaboyer, B., Jevremović, D., et al. 2008, ApJS, 178, 89
Dumusque, X., Bonomo, A. S., Haywood, R. D., et al. 2014, ApJ, 789, 154 Fabrycky, D., \& Tremaine, S. 2007, ApJ, 669, 1298

Foreman-Mackey, D., Montet, B. T., Hogg, D. W., et al. 2015, ApJ, 806, 215 Fressin, F., Torres, G., Charbonneau, D., et al. 2013, ApJ, 766, 81

Han, E., Wang, S. X., Wright, J. T., et al. 2014, PASP, 126, 827

Hansen, B. M., \& Murray, N. 2012, ApJ, 751, 158

Howell, S. B., Sobeck, C., Haas, M., et al. 2014, PASP, 126, 398

Ida, S., \& Lin, D. 2008, ApJ, 673, 487

Kipping, D. M. 2010, MNRAS, 408, 1758

Kipping, D. M. 2013, MNRAS, 434, L51

Kley, W., \& Nelson, R. 2012, ARA\&A, 50, 211

Kurucz, R. 1993, R. Kurucz CD-ROM No. 13. (Cambridge, Mass.: Smithsonian Astrophysical Observatory), 13

Lecavelier des Étangs, A. 2007, A\&A, 461, 1185

Lissauer, J. J., Jontof-Hutter, D., Rowe, J. F., et al. 2013, ApJ, 770, 131

Liu, S.-F., Hori, Y., Lin, D., \& Asphaug, E. 2015, ApJ, 812, 164

Lopez, E. D., \& Fortney, J. J. 2014, ApJ, 792, 1

Malavolta, L., Borsato, L., Granata, V., et al. 2017, ApJ, 153, 224

Marcy, G. W., Isaacson, H., Howard, A. W., et al. 2014, ApJS, 210, 20

Mayor, M., Pepe, F., Queloz, D., et al. 2003, The Messenger, 114, 20

McNeil, D., \& Nelson, R. 2010, MNRAS, 401, 1691

Montet, B. T., Morton, T. D., Foreman-Mackey, D., et al. 2015, ApJ, 809, 25

Munari, U., Henden, A., Frigo, A., et al. 2014, ApJ, 148, 81

Nissen, P. E. 2015, A\&A, 579, A52

Owen, J. E., \& Jackson, A. P. 2012, MNRAS, 425, 2931

Pepe, F., Mayor, M., Rupprecht, G., et al. 2002, The Messenger, 110, 9

Pollack, J. B., Hubickyj, O., Bodenheimer, P., et al. 1996, Icarus, 124, 62

Pope, B. J., Parviainen, H., \& Aigrain, S. 2016, MNRAS, 461, 3399

Queloz, D., Mayor, M., Weber, L., et al. 2000, A\&A, 354, 99

Santerne, A., Díaz, R., Almenara, J.-M., et al. 2015, MNRAS, 451, 2337

Santos, N. C., Sousa, S. G., Mortier, A., et al. 2013, A\&A, 556, A150

Santos, N. C., Adibekyan, V., Mordasini, C., et al. 2015, A\&A, 580, L13

Schlichting, H. E. 2014, ApJ, 795, L15

Sinukoff, E., Howard, A. W., Petigura, E. A., et al. 2016, ApJ, 827, 78

Sneden, C. 1973, ApJ, 184, 839

Sousa, S. G., Santos, N. C., Israelian, G., Mayor, M., \& Udry, S. 2011, A\&A, 533, A141

Southworth, J. 2008, MNRAS, 386, 1644

Stone, R. C. 1989, AJ, 97, 1227

Tsantaki, M., Sousa, S. G., Adibekyan, V. Z., et al. 2013, A\&A, 555, A150

Tucci Maia, M., Ramírez, I., Meléndez, J., et al. 2016, A\&A, 590, A32

Vanderburg, A., \& Johnson, J. A. 2014, PASP, 126, 948

Vanderburg, A., Latham, D. W., Buchhave, L. A., et al. 2016, ApJS, 222, 14

Weiss, L. M., \& Marcy, G. W. 2014, ApJ, 783, L6

Zeng, L., \& Sasselov, D. 2013, PASP, 125, 227

Zeng, L., \& Sasselov, D. 2014, ApJ, 784, 96 


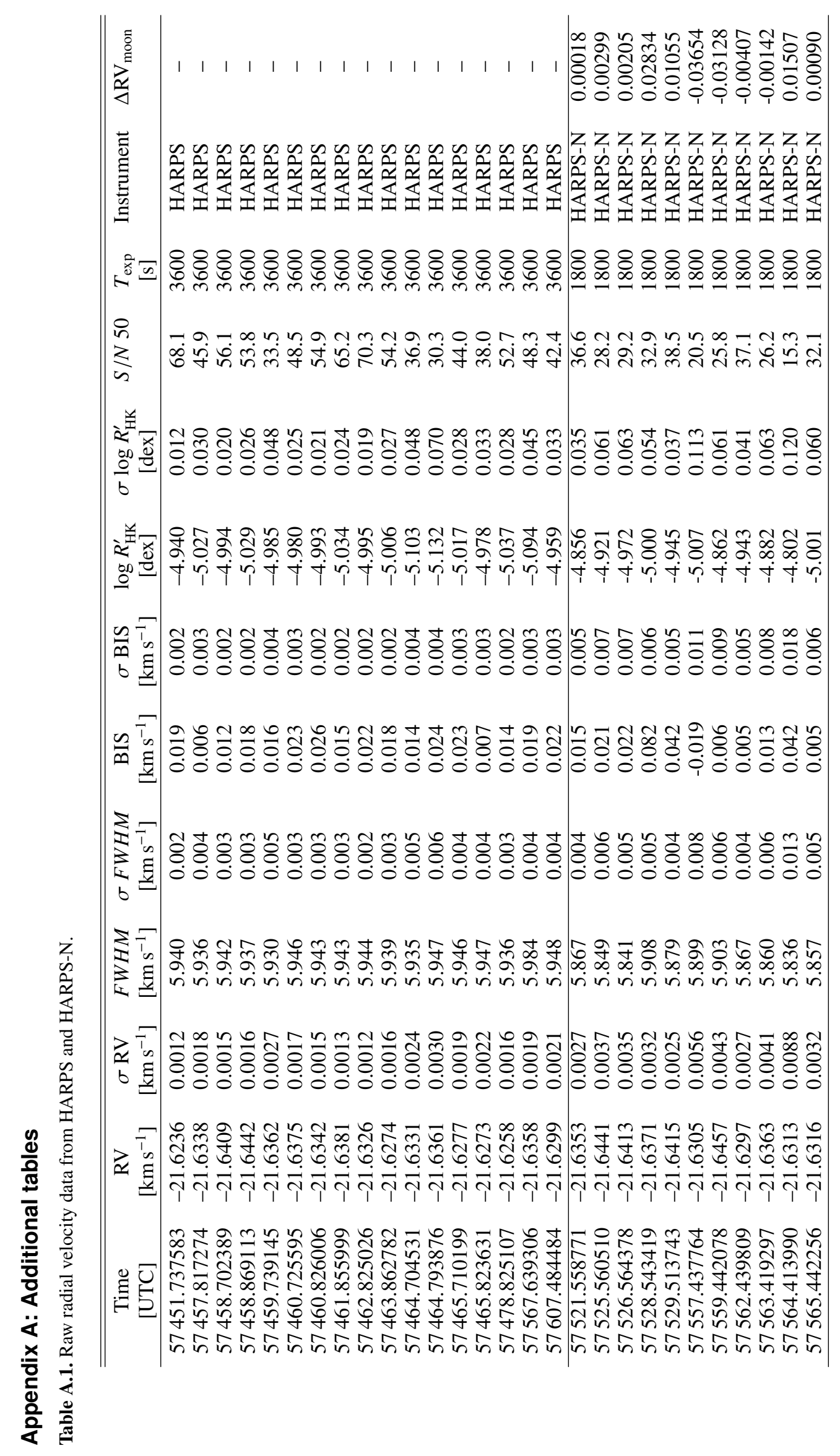


Table A.2. Stellar abundances.

\begin{tabular}{cllccl}
\hline \hline Elem & {$[\mathrm{X} / \mathrm{H}]$} & Error & Elem & {$[\mathrm{X} / \mathrm{H}]$} & Error \\
\hline $\mathrm{O}$ & 0.009 & 0.080 & $\mathrm{Cu}$ & -0.305 & 0.048 \\
$\mathrm{Na}$ I & -0.334 & 0.046 & $\mathrm{Zn} \mathrm{II}$ & -0.323 & 0.101 \\
$\mathrm{Mg}$ I & -0.274 & 0.060 & $\mathrm{Sr}$ & -0.338 & 0.050 \\
$\mathrm{Al} \mathrm{I}$ & -0.157 & 0.035 & $\mathrm{Y} \mathrm{II}$ & -0.425 & 0.093 \\
$\mathrm{Si} \mathrm{I}$ & -0.260 & 0.039 & $\mathrm{Zr}$ & -0.290 & 0.080 \\
$\mathrm{Ca}$ I & -0.223 & 0.062 & $\mathrm{Ba} \mathrm{II}$ & -0.452 & 0.010 \\
$\mathrm{Sc} \mathrm{II}$ & -0.276 & 0.064 & $\mathrm{Ce} \mathrm{I}$ & -0.131 & 0.327 \\
$\mathrm{Ti}$ & -0.205 & 0.092 & $\mathrm{Mg} / \mathrm{Si}$ & 1.191 & - \\
$\mathrm{Cr}$ & -0.279 & 0.074 & $\mathrm{O} / \mathrm{Fe}$ & 0.35 & 0.08 \\
$\mathrm{Mn} \mathrm{I}$ & -0.331 & 0.077 & $A(\mathrm{Li})$ & $<0.2$ & - \\
$\mathrm{Co} \mathrm{I}$ & -0.272 & 0.031 & - & & \\
\hline
\end{tabular}

Table A.3. List of free parameters used in the PASTIS analysis of the light curves, radial velocities and SED with their associated prior and posterior distribution.

\begin{tabular}{|c|c|c|}
\hline Parameter & Prior & Posterior \\
\hline \multicolumn{3}{|l|}{ Orbital parameters } \\
\hline $\begin{array}{l}\text { Orbital period } P[\mathrm{~d}] \\
\text { Epoch of first transit } T_{0}\left[\mathrm{BJD}_{\mathrm{TDB}}\right]-2.45 \times 10^{6} \\
\text { Orbital eccentricity } e \\
\text { Argument of periastron } \omega\left[^{\circ}\right] \\
\text { Inclination } i\left[^{\circ}\right]\end{array}$ & $\begin{array}{c}\mathcal{U}(13.8 ; 13.9) \\
\mathcal{U}(7275.2 ; 7275.5) \\
\beta(0.867 ; 3.03) \\
\mathcal{U}(0 ; 360) \\
\mathcal{S}(70 ; 90)\end{array}$ & $\begin{array}{c}13.86375 \pm 2.6 \times 10^{-4} \\
7275.32991 \pm 6.3 \times 10^{-4} \\
0.091 \pm 0.089 \\
90_{-64}^{+180} \\
89.35_{-0.24}^{+0.41}\end{array}$ \\
\hline \multicolumn{3}{|l|}{ Planetary parameters } \\
\hline $\begin{array}{l}\text { Radial velocity amplitude } K\left[\mathrm{~m} \mathrm{~s}^{-1}\right] \\
\text { Planet-to-star radius ratio } k_{r}\end{array}$ & $\begin{array}{l}\mathcal{U}(0 ; 1000) \\
\mathcal{U}(0 ; 1)\end{array}$ & $\begin{array}{c}5.5 \pm 1.1 \\
0.0333 \pm 6.6 \times 10^{-4}\end{array}$ \\
\hline \multicolumn{3}{|l|}{ Stellar parameters } \\
\hline $\begin{array}{l}\text { Effective temperature } T_{\text {eff }}[\mathrm{K}] \\
\text { Surface gravity } \log g\left[\mathrm{~g} \mathrm{~cm}^{-2}\right] \\
\text { Iron abundance }[\mathrm{Fe} / \mathrm{H}][\mathrm{dex}] \\
\text { Reddening } E(B-V)[\mathrm{mag}] \\
\text { Systemic radial velocity } v_{0}\left[\mathrm{~km} \mathrm{~s}^{-1}\right] \\
\text { Distance to Earth } d[\mathrm{pc}]\end{array}$ & $\begin{array}{c}\mathcal{N}(4960: 60) \\
\mathcal{N}(4.58 ; 0.13) \\
\mathcal{N}(-0.34 ; 0.03) \\
\mathcal{U}(0 ; 1) \\
\mathcal{U}(-25,-15) \\
\mathcal{P}(2 ; 10 ; 1000)\end{array}$ & $\begin{array}{c}5010 \pm 50 \\
4.60 \pm 0.03 \\
-0.34 \pm 0.03 \\
0.019_{-0.013}^{+0.019} \\
-21.6331 \pm 9 \times 10^{-4} \\
118.0 \pm 3.6\end{array}$ \\
\hline \multicolumn{3}{|l|}{ Instrumental parameters } \\
\hline $\begin{array}{l}\text { HARPS radial velocity jitter }\left[\mathrm{m} \mathrm{s}^{-1}\right] \\
\text { HARPS-N radial velocity jitter }\left[\mathrm{m} \mathrm{s}^{-1}\right] \\
\text { HARPS - HARPS-N radial velocity offset }\left[\mathrm{m} \mathrm{s}^{-1}\right] \\
\text { SED jitter [mag] } \\
\text { K2 jitter [ppm] } \\
\text { K2 contamination [ppt] }\end{array}$ & $\begin{array}{c}\mathcal{U}(0 ; 1000) \\
\mathcal{U}(0 ; 1000) \\
\mathcal{U}(-100 ; 100) \\
\mathcal{U}(0 ; 1) \\
\mathcal{U}(0 ; 10000) \\
\mathcal{N}_{\mathcal{U}}(0 ; 5 ; 0 ; 100)\end{array}$ & $\begin{array}{c}3.1 \pm 1.0 \\
3.2 \pm 2.3 \\
-4.2 \pm 1.8 \\
0.021 \pm 0.019 \\
41.2 \pm 4.6 \\
3.4_{-2.4}^{+3.6}\end{array}$ \\
\hline
\end{tabular}

Notes. $\mathcal{N}\left(\mu ; \sigma^{2}\right)$ is a normal distribution with mean $\mu$ and width $\sigma^{2}, \mathcal{U}(a ; b)$ is a uniform distribution between $a$ and $b, \mathcal{N}_{\mathcal{U}}\left(\mu ; \sigma^{2}, a, b\right)$ is a normal distribution with mean $\mu$ and width $\sigma^{2}$ multiplied with a uniform distribution between $a$ and $b, \mathcal{S}(a, b)$ is a sine distribution between $a$ and $b, \beta(a ; b)$ is a Beta distribution with parameters $a$ and $b$, and $\mathcal{P}(n ; a ; b)$ is a power-law distribution of exponent $n$ between $a$ and $b$. The choice of prior for the orbital eccentricity is described in Kipping (2013). 